EGU21-4904, updated on 05 May 2021

https://doi.org/10.5194/egusphere-egu21-4904

EGU General Assembly 2021

(c) Author(s) 2021. This work is distributed under

the Creative Commons Attribution 4.0 License.

\title{
Quantifying the Effects of Urbanization on Floods for two adjacent basins in Shenzhen and Hong Kong, China.
}

\author{
Dandan Liu ${ }^{1,2}$, Yiheng Chen ${ }^{1,3}$, Jinhui Huang ${ }^{1}$, and Xiaogang Shi ${ }^{2}$ \\ ${ }^{1}$ Nankai University, Tianjin, China \\ ${ }^{2}$ University of Glasgow, Glasgow, UK \\ ${ }^{3}$ University of Bristol, Bristol, UK
}

With the accelerating urbanization in developing countries, the threats of extreme rainfall and flood events are increasing. The impact of flood disasters severely threatens the safety of cities with a huge population. In order to quantitatively study the impact of urbanization development on urban floods, the hydrological characteristics of two adjacent basins will be analyzed and compared in this study -- Shenzhen, China with rapid urbanization in the past 40 years, and Hong Kong, China that was already urbanized.

The methods of this study mainly include the following two points. Firstly, in order to clarify the urbanization development process of the study regions, the geospatial database of surface impervious area of two adjacent basins from 1986 to 2018 was obtained in this study. In addition, this study intends to predict the impervious area of the study area in the future years through urban planning. The prediction method is based on adaptive cell deep learning analysis method. Secondly, in order to simulate the waterlogging situation in the two regions, this study intends to select specific flood events to establish and calibrate the SWMM model. By changing the impervious area of the two regions, hydrological parameters such as surface runoff and sensitivity under different scenarios can be obtained.

After model simulation, we will finally analyze the simulation results as follows: Firstly, the variation of runoff and flood peak with impervious area will be analyzed; Secondly, by comparing the simulation results of the two regions, the sensitivity of flood events to urbanization development will be evaluated; Finally, according to the predicted simulation results, the flood situation in the study area will be evaluated in the future years, which has a certain guiding significance for urban flood prevention. 